\title{
Efeito de vespas não-polinizadoras sobre o mutualismo Ficus - vespas de figos
}

\author{
Larissa G. Elias, Vanessa T. do Ó, Fernado H. A. Farache \& Rodrigo A. S. Pereira
}

Depto. de Biologia, Faculdade de Filosofia, Ciências e Letras de Ribeirão Preto, Av. Bandeirantes 3900, 14040-901 Ribeirão Preto, São Paulo, Brasil. (raspereira@ffclrp.usp.br)

\begin{abstract}
Effect of non-pollinating fig wasps over fig-fig wasp mutualism. Mutualism is the name given to interspecific interactions which result in benefit for all partners involved. However, such cooperation is open to opportunistic strategies: individuals that extract the benefit from the partner, but do not offer any benefit in exchange. The fig-fig wasp interaction is an appropriate case to study mutualistic interactions and opportunistic strategies (parasites of mutualism). Plants of the genus Ficus maintain a mutualistic interaction with tiny pollinating wasps (Agaonidae) and are exploited by other non-pollinating fig wasp species. This study aimed to assess the effect of non-pollinating wasps over the mutualistic relation between Ficus citrifolia (Moraceae) and its pollinator Pegoscapus tonduzi Grandi, 1919 (Hymenoptera, Agaonidae). We compared both seed (female plant function) and pollinating female (male plant function) production rates among samples of high- and low-infested figs by non-pollinating wasps, sampled in three Brazilian cities, Londrina (State of Paraná), Campinas and Ribeirão Preto (state of São Paulo), Brazil. Our results have shown a negative impact over both female and male floral reproductive components. This effect was higher on the male plant component (production of pollinating females). Pollinator production was approximately seven times lower in infested figs, whereas seed production was 1.5 times lower in those figs. We discuss hypotheses about mutualism stability with the occurrence of opportunistic species.
\end{abstract}

KEYWORDS. Competition, insect-plant interaction, Hymenoptera, Moraceae, pollination.

RESUMO. Relações ecológicas interespecíficas, que resultam em benefício para todos os organismos participantes, são conhecidas como mutualismo. No entanto, tal cooperação abre espaço para o surgimento de estratégias oportunistas (ou de trapaça), representadas por indivíduos parasitas do mutualismo, que recebem o benefício de um dos parceiros sem oferecer nada em troca. A interação figueiras vespas - de - figo é um sistema adequado para o estudo do mutualismo e de estratégias oportunistas (parasitas de mutualismos). Representantes do gênero Ficus (Moraceae) apresentam uma relação mutualística com pequenas vespas polinizadoras (Agaonidae) e são explorados por outras espécies de vespas não-polinizadoras. Esse trabalho teve como objetivo avaliar o impacto das vespas nãopolinizadoras sobre o mutualismo Ficus citrifolia e suas vespas polinizadoras, Pegoscapus tonduzi Grandi, 1919. Para tal, foi comparada a produção de aquênios (função feminina) e de fêmeas da espécie polinizadora (função masculina) entre amostras de sicônios altamente infestados e pouco infestados por vespas não-polinizadoras, coletadas nos municípios de Londrina (Paraná), Campinas e Ribeirão Preto (São Paulo), Brasil. Nossos resultados apontaram que as vespas não-polinizadoras exercem impacto negativo nos componentes feminino e masculino da planta, sendo maior no masculino. A produção de vespas polinizadoras foi cerca de sete vezes menor nos figos infestados, ao passo que a produção de aquênios foi 1,5 vez menor nesses mesmos figos. Hipóteses sobre a estabilidade do mutualismo na presença das espécies oportunistas são discutidas.

PALAVRAS-CHAVE. Competição, interação inseto-planta, Hymenoptera, Moraceae, polinização.

Relações ecológicas interespecíficas, que resultam em benefício para todos os organismos participantes, são conhecidas como mutualismo (BEGON et al., 1996). No entanto, tal cooperação abre espaço para o surgimento de estratégias oportunistas, representadas por indivíduos parasitas do mutualismo, que recebem o benefício de um dos parceiros sem oferecer nada em troca (Yu, 2001). A interação figueiras - vespas de figo representa um sistema adequado para o estudo do mutualismo, já que as plantas do gênero Ficus (Moraceae) apresentam uma relação mutualística, espécie-específica, com pequenas vespas polinizadoras da família Agaonidae (Hymenoptera), que utilizam os ovários das flores femininas para depositar seus ovos. As plantas também são exploradas por espécies parasitas do mutualismo, em geral da superfamília Chalcidoidea (Weiblen, 2002).

A polinização das flores de Ficus é realizada exclusivamente pelas fêmeas de Agaonidae e o ciclo de desenvolvimento do sicônio (inflorescência de Ficus) e das vespas pode ser dividido em cinco fases (GALIL \& EIsIKowitch, 1968). Na fase A (pré-feminina), as flores do sicônio ainda estão imaturas, enquanto que na fase B (feminina), fêmeas polinizadoras (fundadoras), fecundadas e carregadas de pólen, são atraídas por substâncias voláteis liberadas pelos sicônios receptivos (HossaerT-MCKey et al., 1994). As vespas adentram a inflorescência através de uma abertura denominada ostíolo, polinizam as flores femininas e depositam ovos em algumas delas. As flores que recebem ovos se transformam em galhas, nas quais a prole de vespas se desenvolve no lugar dos frutos (aquênios), durante a fase C (interfloral). Na fase D (masculina), pouco antes do amadurecimento do sicônio, a prole de vespas completa seu desenvolvimento. Os primeiros a emergir são os machos, que são apteros e têm suas atividades restritas ao interior do sincônio. Os machos localizam e copulam as fêmeas, que ainda estão em suas galhas. $\mathrm{Na}$ seqüência, escavam, com suas mandíbulas, um túnel na parede da inflorescência, por onde as fêmeas sairão. As fêmeas fecundadas emergem, coletam o pólen (em algumas espécies, as fêmeas não coletam o pólen, transportando-o passivamente sobre o corpo) e abandonam o sicônio em busca de uma árvore com sicônios receptivos (Bronstein, 1992). Posteriormente, 
os sicônios completam seu amadurecimento tornandose atrativos para várias espécies de vertebrados frugívoros que atuam como dispersores (JANZEN, 1978).

As vespas não-polinizadoras utilizam o figo para desenvolver sua progênie, mas não realizam polinização, uma vez que depositam seus ovos pelo lado externo, inserindo seu longo ovipositor através da parede do figo (Weiblen, 2002). A biologia dessas vespas é variada, podendo ser galhadoras, inquilinas (cleptoparasitas) ou parasitóides (ABdurahiman \& Joseph, 1978; Weiblen, 2002). As parasitas galhadoras são fitófagas e capazes de induzir galhas nas flores femininas do figo, competindo com as polinizadoras por sítios de oviposição (WEST \& HerRe, 1994; West et al., 1996; Yu, 2001). As vespas inquilinas são fitófagas, mas não induzem a formação de galhas; elas ovipõem em galhas induzidas por outra espécie e se alimentam do tecido vegetal, matando por inanição a larva da espécie galhadora. As espécies parasitóides, por sua vez, desenvolvem suas proles parasitando as larvas de espécies fitófagas (KeRDELhuÉ \& Rasplus, 1996; WeIBLEN, 2002).

As vespas não-polinizadoras afetam negativamente a produção de frutos (função feminina da planta), ovipositando em ovários que poderiam se desenvolver em frutos, além de prejudicarem a produção de vespas polinizadoras dispersoras de pólen (função masculina da planta) (WeSt \& HeRRE, 1994; KeRDELHUÉ \& Rasplus, 1996; West et al., 1996; PEREIRA, 1998). Os efeitos sobre o mutualismo variam de acordo com a ecologia e a biologia das vespas não-polinizadoras envolvidas (BRONSTEIN, 1992): as espécies galhadoras interferem tanto na produção de frutos quanto na de polinizadoras, devido à competição por sítios de oviposição. As espécies inquilinas e parasitóides, por sua vez, apresentam maior efeito negativo sobre a prole de vespas polinizadoras.

Esse trabalho tem como objetivo avaliar o impacto das vespas não-polinizadoras sobre o mutualismo entre Ficus citrifolia e sua vespa polinizadora, Pegoscapus tonduzi Grandi, 1919. Para tal, foi comparada a produção de aquênios (função feminina) e de fêmeas da espécie polinizadora (função masculina) entre amostras de sicônios altamente infestados e pouco infestados por vespas não-polinizadoras.

\section{MATERIAL E MÉTODOS}

$\mathrm{Na}$ fase de emergência da prole de vespas foram coletados cerca de 20 a 50 sicônios por árvore em 10 indivíduos de Ficus citrifolia. Cinco amostras pouco infestadas por vespas não-polinizadoras (menos de 10 vespas por sicônio), foram selecionadas de coletas realizadas nas proximidades do campus da Universidade Estadual de Campinas (UNICAMP), Campinas, SP ( $\left.22^{\circ} 54^{\prime} \mathrm{S} ; 47^{\circ} 03^{\prime} \mathrm{W}\right)$, no período de abril a junho dos anos de 1998 e 1999. Três safras altamente infestadas por vespas não-polinizadoras foram selecionadas de coletas no campus da Universidade de São Paulo (USP), Ribeirão Preto, SP ( $21^{\circ} 10^{\prime}$ S; $\left.47^{\circ} 48^{\prime} \mathrm{W}\right)$, no mês de maio de $2003 \mathrm{e}$ no período de maio a junho de 2005, e outras duas no campus da Universidade Estadual de Londrina, Londrina, PR ( $\left.23^{\circ} 23^{\prime} \mathrm{S} ; 51^{\circ} 11^{\prime} \mathrm{W}\right)$ no período de agosto a outubro de 1992 (Tab. I).

Os figos foram individualizados em frascos plásticos e mantidos em laboratório por aproximadamente 48 horas para emergência das vespas. Posteriormente, $\mathrm{o}$ material foi congelado e preservado em álcool até a triagem. Os exemplares de cada figo foram identificados em nível genérico, quantificados e sexados. Os aquênios foram contados em uma seção equivalente a $1 / 4$ do figo e o número total por figo foi estimado multiplicando-se por quatro o valor quantificado na seção.

A produção média de fêmeas de polinizadoras e de aquênios por figo nos dois grupos (pouco infestados e muito infestados) foi comparada por teste $t$ (ZAR, 1996). Para corrigir o efeito do tamanho do sicônio (sicônios maiores apresentariam maior número de flores femininas e, conseqüentemente, mais aquênios e vespas), o número de vespas polinizadoras e aquênios foi dividido pelo diâmetro do figo. Assim, as estimativas do número de polinizadoras e aquênios foram representadas pela abundância por centímetro de diâmetro de figo. Foram analisados 137 sicônios pouco infestados e 158 sicônios muito infestados.

\section{RESULTADOS}

Foram analizados 137 sicônios pouco infestados, nos quais encontramos a espécie polinizadora e três outras do gênero Idarnes Walker 1843 (Agaonidae sensu

Tabela I. Número (média \pm DP) de aquênios e vespas polinizadoras e não-polinizadoras por sicônio provenientes de amostras de Ficus citrifolia pouco e altamente infestadas. (n, número de sicônios amostrados).

\begin{tabular}{|c|c|c|c|c|c|c|c|}
\hline \multirow[t]{2}{*}{ Grupo } & \multirow[t]{2}{*}{ Amostra } & \multirow{2}{*}{$\begin{array}{l}\text { Mês de } \\
\text { coleta }\end{array}$} & \multirow[t]{2}{*}{$\mathrm{n}$} & \multirow[t]{2}{*}{ Aquênios } & \multicolumn{2}{|c|}{ P. tonduzi } & \multirow[t]{2}{*}{ Não-polinizadoras } \\
\hline & & & & & Fêmeas & Machos & \\
\hline \multirow[t]{5}{*}{ Pouco infestado } & Campinas 1 & maio & 18 & $285,1 \pm 32,5$ & $252,7 \pm 51,2$ & $26,2 \pm 19,9$ & $0,2 \pm 0,7$ \\
\hline & Campinas 2 & abril & 49 & $171,1 \pm 36,0$ & $252,4 \pm 64,1$ & $18,2 \pm 11,1$ & 0 \\
\hline & Campinas 3 & abril & 18 & $254,6 \pm 32,7$ & $200,5 \pm 28,0$ & $20,1 \pm 9,7$ & $0,5 \pm 1,7$ \\
\hline & Campinas 4 & abril & 24 & $164,0 \pm 46,4$ & $223,6 \pm 35,1$ & $14,6 \pm 8,5$ & $0,6 \pm 1,7$ \\
\hline & Campinas 5 & abril & 28 & $150,1 \pm 38,4$ & $192,8 \pm 24,4$ & $13,8 \pm 9,1$ & 0 \\
\hline \multirow[t]{5}{*}{ Muito infestado } & Londrina 1 & outubro & 38 & $142,8 \pm 68,8$ & $7,6 \pm 9,1$ & $4,7 \pm 5,5$ & $102,9 \pm 35,4$ \\
\hline & Londrina 2 & outubro & 44 & $171,7 \pm 56,2$ & $36,5 \pm 39,9$ & $4,7 \pm 4,0$ & $62,0 \pm 26,7$ \\
\hline & R. Preto 1 & maio & 26 & $146,3 \pm 31,5$ & $79,2 \pm 35,8$ & $10,4 \pm 9,6$ & $100,7 \pm 29,6$ \\
\hline & R. Preto 2 & junho & 25 & $107,9 \pm 42,4$ & $28,0 \pm 26,8$ & $3,5 \pm 4,6$ & $41,4 \pm 19,2$ \\
\hline & R. Preto 3 & junho & 25 & $137,7 \pm 48,4$ & $21,8 \pm 23,1$ & $3,4 \pm 3,7$ & $26,0 \pm 15,1$ \\
\hline
\end{tabular}


Boucek, 1993), uma espécie de Physothorax Mayr 1885 (Torymidae) e uma de Eurytoma Illiger, 1807 (Eurytomidae). Nas amostras altamente infestadas, representadas por 158 sicônios analizados, ocorreram a espécie polinizadora e representantes dos gêneros Idarnes (quatro espécies), Torymus Dalman, 1820 (Torymidae) (uma espécie), Physotorax (duas espécies), Heterandrium Mayr 1885 (Pteromalidae) (duas espécies), Eurytoma (duas espécies) e Aepocerus Mayr, 1885 (Pteromalidae) (duas espécies) (Tab. II). A tabela I resume as características de cada amostra analisada.

$\mathrm{O}$ grupo de sicônios pouco infestados apresentou densidades ( $\mathrm{n}^{\circ}$ por centímetro de diâmetro de figo) de fêmeas polinizadoras e de aquênios significativamente maiores que o grupo de sicônios infestados (fêmeas polinizadoras: $t_{155}=11,9 ; p<10^{-22}$; aquênios: $t_{266}=3,9 ; p<$ $10^{-3}$; Fig. 1).
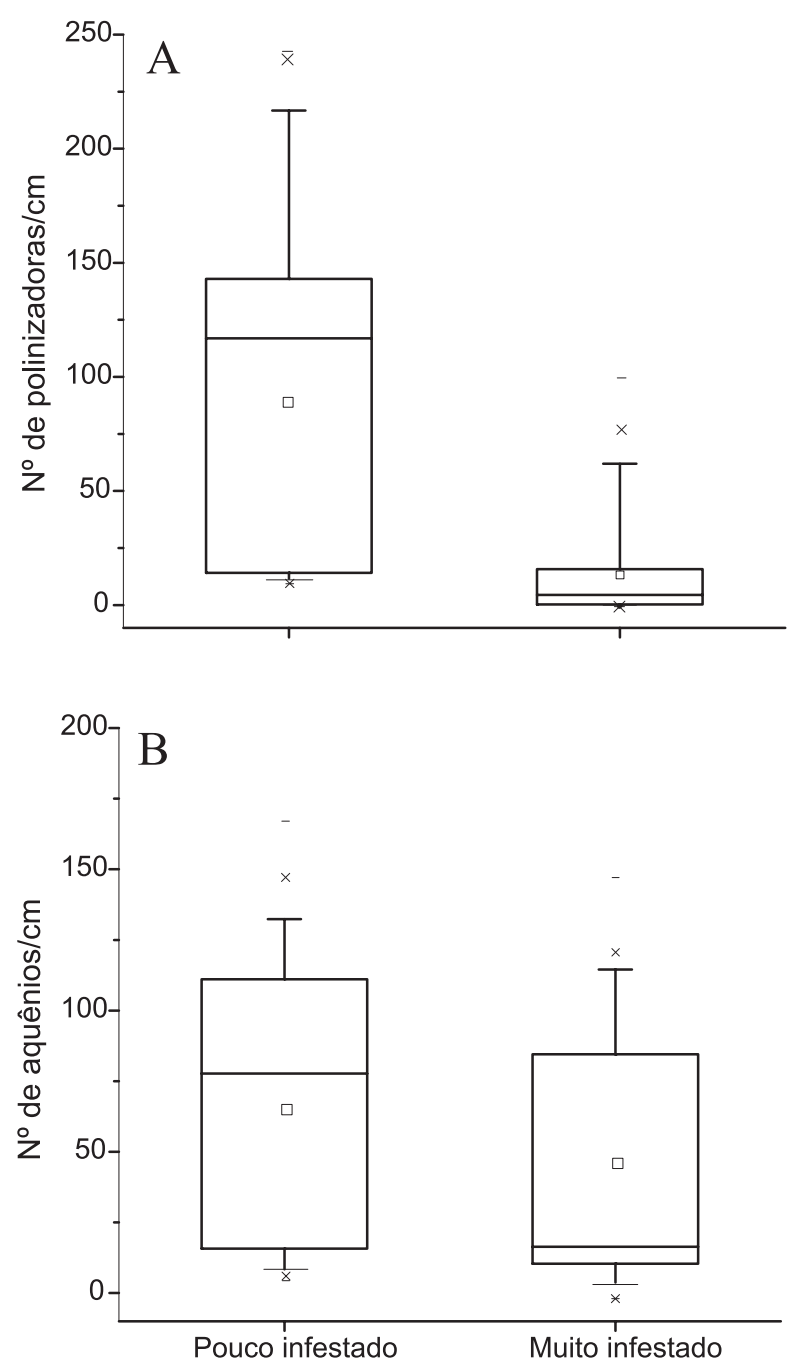

Fig. 1. Densidade ( ${ }^{\circ}$ por centímetro de diâmetro de figo) de (A) fêmeas de vespas polinizadoras Pegoscapus tonduzi Grandi, 1919 e (B) de aquênios nas amostras pouco $(n=137$ sicônios $)$ e muito infestadas ( $\mathrm{n}=158$ sicônios). O retângulo delimita $50 \%$ do número de observações e o traço central representa a mediana. As barras indicam os valores do $5^{\circ}$ e do $95^{\circ}$ percentis. Os símbolos abaixo das barras indicam o $0^{\circ}$ e o $1^{\circ}$ percentis e os acima o $99^{\circ}$ e o $100^{\circ}$ percentis. O símbolo quadrado representa a média dos valores.
Tabela II. Número (média por sicônio \pm DP) de indivíduos por espécie de vespa associada aos grupos de sicônios pouco e muito infestados de $F$. citrifolia $(n=137$, pouco infestados; $n=158$, muito infestados).

\begin{tabular}{lcc}
\hline Espécie & Pouco infestados & Muito infestados \\
\hline Pegoscapus tonduzi & $246,3 \pm 61,9$ & $38,1 \pm 40,3$ \\
Aepocerus sp. 1 & 0 & $0,6 \pm 1,7$ \\
Aepocerus sp. 2 & 0 & $0,01 \pm 0,1$ \\
Eurytoma butcheri & $0,02 \pm 0,3$ & $0,3 \pm 1,0$ \\
Eurytoma sp. 2 & 0 & $0,006 \pm 0,8$ \\
Heterandrium sp. 1 & 0 & $0,2 \pm 0,9$ \\
Heterandrium sp. 2 & 0 & $0,2 \pm 1,2$ \\
Idarnes carme & $0,01 \pm 0,2$ & $42,5 \pm 28,9$ \\
Idarnes flavicollis & $0,08 \pm 0,6$ & $17 \pm 19,4$ \\
Idarnes incerta & $0,08 \pm 0,6$ & $0,2 \pm 0,6$ \\
Physothorax sp. 1 & $0,01 \pm 0,1$ & $2,7 \pm 4,3$ \\
Physothorax sp. 2 & 0 & $0,1 \pm 0,4$ \\
Torymus sp. & 0 & $12,4 \pm 12,9$ \\
\hline
\end{tabular}

\section{DISCUSSÃO}

Os resultados apontaram que vespas nãopolinizadoras associadas a $F$. citrifolia exercem um impacto negativo nos componentes masculino e feminino das plantas (produção de fêmeas das vespas polinizadoras e aquênios, respectivamente). O impacto negativo no número de vespas polinizadoras pode ser resultante da competição com espécies fitófagas pelos mesmos sítios de oviposição (flores femininas) ou da ação direta de espécies parasitóides e inquilinas. A redução no número de aquênios nos figos infestados é, possivelmente, resultado da utilização de flores femininas por espécies não-polinizadoras fitófagas, tais como alguns representantes do gênero Idarnes (PEREIRA, 1998).

O impacto negativo das vespas não-polinizadoras foi maior no componente masculino das plantas, já que a produção de vespas polinizadoras foi cerca de sete vezes menor nos figos mais infestados, e a produção de aquênios foi 1,5 vez menor nesses mesmos figos. Essa constatação corrobora estudos prévios realizados em espécies de Ficus do Novo e Velho Mundo (WeSt \& Herre, 1994; CoOK \& Power, 1996; Kerdelhué \& Rasplus, 1996; West et al., 1996). O desenvolvimento das vespas e a produção dos aquênios em camadas distintas de ovários possivelmente impedem que o efeito das vespas sobre a produção de aquênios seja mais efetivo (WEST \& Herre, 1994). As vespas associadas às espécies monóicas de Ficus depositam seus ovos preferencialmente nos ovários das flores mais próximas ao lúmen do sicônio e os aquênios são produzidos nas camadas mais profundas (próximas à parede do sicônio) (KJELLbERG et al., 2005). Especula-se que as flores próximas ao lúmen seriam mais adequadas ao desenvolvimento dos insetos, facilitando a emergência dos adultos e o acesso a acasalamentos (AnstetT, 2001). Desta forma, as vespas-de-figo seriam selecionadas a depositar seus ovos nas flores de melhor qualidade e a competição por sítios de oviposição seria mais intensa nos ovários mais próximos ao lúmen do sicônio. Essa hipótese é apoiada pela constatação, em $F$. burtt-davyi, de que os ovários das camadas mais 
profundas (próximas à parede do sicônio) são explorados pelas vespas em situações de alta infestação (NEFDT \& COMPTON, 1996).

Além do impacto negativo imediato nos componentes reprodutivos das plantas, as vespas nãopolinizadoras podem desestabilizar, a longo prazo, o mutualismo Ficus - vespas-de-figo, gerando uma limitação de polinização devido à redução na produção de fêmeas polinizadoras, e à menor dispersão de diásporos, relacionada à redução na produção de aquênios. Desta forma, seria esperado que a seleção natural favorecesse mecanismos que limitassem o desenvolvimento das espécies parasitas do mutualismo nas figueiras. No entanto, adaptações relacionadas à exclusão das vespas não-polinizadoras provavelmente atuariam negativamente sobre as polinizadoras (Yu, 2001). Além disso, a planta provavelmente não apresenta mecanismos para distinguir entre ovos ou larvas de nãopolinizadoras e polinizadoras (BRoNSTEIN, 1992). A manutenção do mutualismo na presença de vespas nãopolinizadoras seria possível, ainda, pela ocorrência de artrópodos (aranhas e formigas) que as predam durante o processo de oviposição, mantendo a densidade populacional dessas espécies em um nível que não desestabilize o mutualismo (COMPTON \& ROBERTSON, 1988).

Os resultados apresentados não apóiam observações anteriores de maiores infestações por nãopolinizadoras em fragmentos florestais e áreas alteradas pela ação antrópica (WANG et al., 2005; R. A. S. Pereira, obs. pessoal), uma vez que as amostras com baixa infestação (Campinas, SP) são provenientes de áreas urbanizadas com forte ação antrópica. No entanto, as densidades populacionais de $F$. citrifolia foram acentuadamente mais altas nas áreas com maiores infestações (Londrina, PR e Ribeirão Preto, SP). Possíveis efeitos sazonais relacionados aos períodos de coleta são improváveis, uma vez que as amostragens concentraramse entre o início e o final da estação seca. Desta forma, as disponibilidades espacial e temporal de sicônios seriam, potencialmente, fatores determinantes na regulação populacional das espécies polinizadoras e nãopolinizadoras. Estudos detalhados sobre a ecologia destas vespas são necessários para responder tais questões e constituem uma linha aberta para futuros estudos.

Agradecimentos. Este estudo recebeu apoio financeiro da International Foundation for Science (processo\#D/3692-1). A primeira autora recebeu bolsa de Iniciação Científica PIBIC/CNPq. Este estudo foi desenvolvido no Pós-Graduação em Entomologia, FFCLRP - USP.

\section{REFERÊNCIAS BIBLIOGRÁFICAS}

Abdurahiman, U. C. \& Joseph, K. J. 1978. Cleptoparasitism of the fig wasps (Torymidae: Chalcidoidea) in Ficus hispida L. Entomon 3:181-186.

Anstett, M. C. 2001. Unbeatable strategy, constraint and coevolution, or how to resolve evolutionary conflicts: the case of the fig/fig wasp mutualism. Oikos 95:476-484.

Begon, M.; Harper, J. L. \& Townsend, C. R. 1996. Ecology: individuals, population and communities. Oxford, Blackwell Science. 1068p.

Boucek, Z. 1993. The genera of chalcidoid wasp from Ficus fruit in the New World. Journal of Natural History 27:173-217.

BRonstein, J. L. 1992. Seed predator as mutualists: ecology and evolution of the fig pollinator interaction. In: Bernays, E. ed. Insect-Plant interactions. Boca Raton, CRC. p.1-43.

Compton, S. G. \& Robertson, H. G. 1988. Complex interactions between mutualisms: ants tending homopterans protect fig seeds and pollinators. Ecology 69:1302-1305.

СоoK, J. M. \& Power, S. A. 1996. Effects of within-tree flowering asynchrony on the dynamics of seed and wasp production in an Australian fig species. Journal of Biogeography 23:487-493.

GaliL, J. \& EisiKowitch, D. 1968. On the pollination ecology of Ficus sycomorus in east Africa. Ecology 49:259-269.

Hossaert-McKey, M.; Gibernau, M. \& Frey, J. E. 1994. Chemosensory attraction of fig wasps to substances produced by receptive figs. Entomologia Experimentalis et Applicata 70:185-191

JANZEN, D. H. 1978. A bat-generated fig seed shadow in rain forest. Biotropica 10:121.

Kerdelhué, C. \& Rasplus, J. Y. 1996. Non-pollinating Afrotropical fig wasps affect the fig-pollinator mutualism in Ficus within the subgenus Sycomorus. Oikos 75:3-14.

Kuellberg, F.; Jousselin, E.; Hossaert-Mckey, M. \& Rasplus, J. Y. 2005. Biology, Ecology and Evolution of Fig-pollinating Wasps (Chalcidoidea, Agaonidae). In: Raman, A.; Schaefer, C. W. \& Withers, T. M. eds. Biology, ecology and evolution of gall-inducing arthropods. New Hampshire, Science. p.539-571

Nefdt, R. J. C. \& Compton, S. G. 1996. Regulation of seed and pollinator production in the fig-fig wasp mutualism. Journal of Animal Ecology 65:170-182.

Pereira, R. A. S.; Semir, J. \& Menezes Jr., A. O. 2000. Pollination and other biotic interactions in figs of Ficus eximia Schott (Moraceae). Brazilian Journal of Botany 23:217-224.

WANG, R. W.; YANG, C. Y.; ZhaO, G. F. \& YANG, J. X. 2005 Fragmentation effects on diversity of wasp community and its impact on fig/fig wasp interaction in Ficus racemosa L. Journal of Integrative Plant Biology 47:20-26.

Weiblen, G. D. 2002. How to be a fig wasp. Annual Review of Entomology 47:299-330.

West, S. A. \& Herre, E. A. 1994. The ecology of the New World fig-parasitizing wasps Idarnes and implications for the evolution of the fig-pollinator mutualism. Proceedings of the Royal Society of London, Series B, Biological Sciences 258(1351):67-72.

West, S. A.; Herre, E. A.; Windsor, D. M. \& Green, P. R. S. 1996. The ecology and evolution of the New World non-pollinating fig wasps communities. Journal of Biogeography 23:447-458.

Yu, D. W. 2001. Parasites of mutualisms. Biological Journal of the Linnean Society 72:529-546.

ZAR, J. H. 1996. Biostatistical analysis. Upper Saddle River, Prentice Hall. 530p. 